\title{
DAUGAVET CENTERS AND DIRECT SUMS OF BANACH SPACES
}

\author{
TETIANA V. BOSENKO
}

\begin{abstract}
A linear continuous nonzero operator $G: X \rightarrow Y$ is a Daugavet center if every rank-1 operator $T: X \rightarrow Y$ satisfies $\|G+T\|=$ $\|G\|+\|T\|$. We study the case when either $X$ or $Y$ is a sum $X_{1} \oplus_{F} X_{2}$ of two Banach spaces $X_{1}$ and $X_{2}$ by some two-dimensional Banach space $F$. We completely describe the class of those $F$ such that for some spaces $X_{1}$ and $X_{2}$ there exists a Daugavet center acting from $X_{1} \oplus_{F} X_{2}$, and the class of those $F$ such that for some pair of spaces $X_{1}$ and $X_{2}$ there is a Daugavet center acting into $X_{1} \oplus_{F} X_{2}$. We also present several examples of such Daugavet centers.
\end{abstract}

\section{Introduction}

In the present paper we consider real Banach spaces which do not equal $\{0\}$, and denote them $E, X$ or $Y$. A linear continuous nonzero operator $G$ : $X \rightarrow Y$ is called a Daugavet center [3] if every rank-1 operator $T: X \rightarrow Y$ satisfies the equation

$$
\|G+T\|=\|G\|+\|T\| .
$$

Definition 1.1. We say that $X$ is a Daugavet domain if there exists a Daugavet center $G$ : $X \rightarrow Y$ for some $Y$, and is a Daugavet range if there is a Daugavet center $G: E \rightarrow X$ for some $E$.

Throughout this paper $F=\left(\mathbb{R}^{2},\|\cdot\|\right)$ with $\|(1,0)\|=\|(0,1)\|=1$ and

$$
\left\|\left(a_{1}, a_{2}\right)\right\|=\left\|\left(\left|a_{1}\right|,\left|a_{2}\right|\right)\right\|
$$

for every $\left(a_{1}, a_{2}\right) \in F$. For Banach spaces $X_{1}$ and $X_{2}$ their $F$-sum $X_{1} \oplus_{F} X_{2}$ is the space of all pairs $\left(x_{1}, x_{2}\right)$ where $x_{1} \in X_{1}$ and $x_{2} \in X_{2},\left\|\left(x_{1}, x_{2}\right)\right\|:=$ $\left\|\left(\left\|x_{1}\right\|,\left\|x_{2}\right\|\right)\right\|_{F}$.

We introduce the following order on $F:\left(a_{1}, a_{2}\right) \geq\left(b_{1}, b_{2}\right)$ if $a_{1} \geq b_{1}$ and $a_{2} \geq b_{2}$. It follows from (1.2) and a convexity argument that for every $\left(a_{1}, a_{2}\right),\left(b_{1}, b_{2}\right) \in F$ with $\left(\left|a_{1}\right|,\left|a_{2}\right|\right) \leq\left(\left|b_{1}\right|,\left|b_{2}\right|\right)$ the inequality $\left\|\left(a_{1}, a_{2}\right)\right\| \leq$ $\left\|\left(b_{1}, b_{2}\right)\right\|$ holds true. In this partial order $F$ is a Banach lattice [8], so we will use the term "two-dimensional lattice" for $F$ in the sequel.

The problem which we solve in this paper, consists of two parts: first, we characterize the class of those $F$ for which there exist $X_{1}$ and $X_{2}$ such that

2000 Mathematics Subject Classification. Primary 46B04; secondary 46B20, 46B40.

Key words and phrases. Daugavet center, Daugavet property, direct sum of Banach spaces. 
$X_{1} \oplus_{F} X_{2}$ is a Daugavet domain, and secondly, we characterize the class of those $F$ for which there are $X_{1}$ and $X_{2}$ such that $X_{1} \oplus_{F} X_{2}$ is a Daugavet range.

Remark that a Daugavet domain and a Daugavet range are generalizations of a Banach space with the Daugavet property, and this motivates our interest in the subject. A Banach space $X$ is said to have the Daugavet property if the identity operator Id: $X \rightarrow X$ is a Daugavet center. The study of spaces with the Daugavet property is a rapidly developing branch of Banach space theory (see [6], [1], [13], and the most recent developments in [5], [7]). The following classical spaces have the Daugavet property: $C(K)$ where $\mathrm{K}$ is a compact without isolated points [4, $L_{1}(\mu)$ and $L_{\infty}(\mu)$ where $\mu$ has no atoms [9], and many Banach algebras ([14], [15]). Some exotic spaces have the Daugavet property as well, for instance, Talagrand's space ([6], [12]) and Bourgain-Rosenthal's space ([2], [7]).

Let us recall some recent results [3] related to Daugavet centers. If $G$ is a Daugavet center then (1.1) also holds true when $\mathrm{T}$ is a strong RadonNikodým operator, e.g., a weakly compact operator. If $X$ is a Daugavet domain or a Daugavet range then $X$ contains subspaces isomorphic to $\ell_{1}$, is non-reflexive and does not have an unconditional basis (countable or uncountable). One cannot even embed such an $X$ into a space having an unconditional basis or having a representation as unconditional sum of reflexive subspaces. In [10] Popov proves that every isometric embedding of $L_{1}[0,1]$ into itself is a Daugavet center. However, in [3] one can find examples of Daugavet centers which are not isometries.

The present work is inspired by [1]. It was shown in [1] and [6] that if $X_{1}$ and $X_{2}$ have the Daugavet property and $F=\ell_{1}^{(2)}$ or $\ell_{\infty}^{(2)}$ then $X_{1} \oplus_{F} X_{2}$ has the Daugavet property as well. In [1] the authors prove that $X_{1} \oplus_{F} X_{2}$ has the Daugavet property only if $F=\ell_{1}^{(2)}$ or $\ell_{\infty}^{(2)}$. In our paper we generalize these results of [1, but use a new approach to the problem. Surprisingly in both parts of our problem we discover other spaces apart from $F=\ell_{1}^{(2)}$ and $F=\ell_{\infty}^{(2)}$, which satisfy our demands.

Our approach is based on a necessary condition for a general Banach space $X$ to be a Daugavet domain and on a necessary condition for $X$ to be a Daugavet range. We deduce these two conditions in Section 2 (see Definition 2.1, Lemma 2.5 and Definition 2.2, Lemma 2.6) and then we show in Section 3 how they depend on $F$ when $X=X_{1} \oplus_{F} X_{2}$ (see Lemma 3.6 and Lemma 3.7).

In Section 4 we find a rather small class $\mathfrak{N}_{2}$ such that if $X_{1} \oplus_{F} X_{2}$ is a Daugavet range then $F \in \mathfrak{N}_{2}$, and in Section 5 we discover the analogous class $\mathfrak{M}_{2}$ for the case of a Daugavet domain. Then for every $F \in \mathfrak{M}_{2}$ we present an example of a Daugavet center acting from a sum of two Banach spaces by $F$ (see Proposition 6.2), and this solves the first part of our problem. In a very similar way we solve its second part, namely we give examples of Daugavet centers acting into a sum of two Banach spaces by $F$ 
for every $F \in \mathfrak{N}_{2}$ (see Proposition [6.6). The obtained results illustrate that the notions of a Daugavet domain and a Daugavet range do not refer to the same Banach spaces.

Throughout this paper $B_{X}$ denotes the closed unit ball of $X$ and $S_{X}$ denotes its unit sphere. We use the notation

$$
B_{F}^{+}:=\left\{a \in B_{F}: a \geq 0\right\}
$$

for the positive part of the unit ball and

$$
S_{F}^{+}:=\left\{a \in S_{F}: a \geq 0\right\}
$$

for the positive part of the unit sphere of $F$. We denote

$$
S\left(B_{X}, z^{*}, \varepsilon\right):=\left\{x \in B_{X}: z^{*}(x)>1-\varepsilon\right\}
$$

the slice of $B_{X}$ determined by $z^{*} \in S_{X^{*}}$ and $\varepsilon>0$.

$$
S\left(B_{X^{*}}, z, \varepsilon\right)=\left\{x^{*} \in B_{X^{*}}: x^{*}(z)>1-\varepsilon\right\}
$$

denotes the weak* slice of $B_{X^{*}}$ determined by $z \in S_{X}$ and $\varepsilon>0$. For an $x^{*} \in X^{*}$ and a $y \in Y$ the symbol $x^{*} \otimes y$ stands for the operator which acts from $X$ into $Y$ as follows: $\left(x^{*} \otimes y\right)(x)=x^{*}(x) y$.

Finally, let us cite a fact that we frequently use in the sequel.

Theorem 1.2 ([3], Theorem 2.1). For an operator $G: X \rightarrow Y$ with $\|G\|=1$ the following assertions are equivalent:

(i) $G$ is a Daugavet center.

(ii) For every $y_{0} \in S_{Y}, x_{0}^{*} \in S_{X^{*}}$ and $\varepsilon>0$ there is an $x \in S\left(B_{X}, x_{0}^{*}, \varepsilon\right)$ with $\left\|G x+y_{0}\right\|>2-\varepsilon$.

(iii) For every $y_{0} \in S_{Y}, x_{0}^{*} \in S_{X^{*}}$ and $\varepsilon>0$ there is a $y^{*} \in S\left(B_{Y^{*}}, y_{0}, \varepsilon\right)$ with $\left\|G^{*} y^{*}+x_{0}^{*}\right\|>2-\varepsilon$.

\section{BANACH SPACES DENYING The DAUGAVET PROPERTY}

Definition 2.1. We say that $X$ denies the Daugavet property with a set $A \subset S_{X}$ if there is an $\varepsilon>0$ such that for every $y \in A$ there exists an $x^{*} \in S_{X^{*}}$ satisfying

$$
\left\|\mathrm{Id}+x^{*} \otimes y\right\|<2-\varepsilon
$$

Definition 2.2. We say that $X$ star-denies the Daugavet property with a set $A \subset S_{X^{*}}$ if there is an $\varepsilon>0$ such that for every $x^{*} \in A$ there exists a $y \in S_{X}$ satisfying (2.1).

Lemma 2.3. For $A \subset S_{X}$ the following assertions are equivalent:

(i) $X$ denies the Daugavet property with $A$.

(ii) There is an $\varepsilon>0$ such that for every $y \in A$ a functional $x^{*} \in S_{X^{*}}$ may be chosen so that every $x \in S\left(B_{X}, x^{*}, \varepsilon\right)$ fulfills $\|x+y\|<2-\varepsilon$. 
(iii) There is an $\varepsilon>0$ such that for every $y \in A$ a functional $x^{*} \in S_{X^{*}}$ may be chosen so that every $y^{*} \in S\left(B_{X^{*}}, y, \varepsilon\right)$ fulfills $\left\|x^{*}+y^{*}\right\|<$ $2-\varepsilon$.

Proof. $(i) \Rightarrow($ ii $)$ We have that there is an $\varepsilon>0$ such that for every $y \in A$ there exists an $x^{*} \in S_{X^{*}}$ satisfying

$$
\left\|\operatorname{Id}+x^{*} \otimes y\right\|=\sup _{x \in B_{X}}\left\|x+x^{*}(x) y\right\|<2-\varepsilon .
$$

Hence $\left\|x+x^{*}(x) y\right\|<2-\varepsilon$ for every $x \in B_{X}$. Let $x \in S\left(B_{X}, x^{*}, \varepsilon / 2\right)$ then $\|x+y\| \leq\left\|x+x^{*}(x) y\right\|+\left\|y-x^{*}(x) y\right\|<2-\varepsilon+\left|1-x^{*}(x)\right| \cdot\|y\|<2-\varepsilon+\frac{\varepsilon}{2}=2-\frac{\varepsilon}{2}$ which implies $(i i)$.

$(i i) \Rightarrow(i)$ Let $\varepsilon$ and $x^{*}$ be from $(i i)$. It is sufficient to show that $\| x+$ $x^{*}(x) y \| \leq 2-\varepsilon / 2$ for every $x \in B_{X}$. Let $x \in S\left(B_{X}, x^{*}, \varepsilon / 2\right)$ then

$\left\|x+x^{*}(x) y\right\| \leq\|x+y\|+\left\|y-x^{*}(x) y\right\|<2-\varepsilon+\left|1-x^{*}(x)\right| \cdot\|y\|<2-\frac{\varepsilon}{2}$.

Let $x \in S\left(B_{X},-x^{*}, \varepsilon / 2\right)$ then $-x \in S\left(B_{X}, x^{*}, \varepsilon / 2\right)$. Hence $\|x-y\|<2-\varepsilon$ and

$\left\|x+x^{*}(x) y\right\| \leq\|x-y\|+\left\|y+x^{*}(x) y\right\|<2-\varepsilon+\left|1+x^{*}(x)\right| \cdot\|y\|<2-\frac{\varepsilon}{2}$.

Finally, let $x \in B_{X} \backslash\left(S\left(B_{X}, x^{*}, \varepsilon / 2\right) \cup S\left(B_{X},-x^{*}, \varepsilon / 2\right)\right)$ then

$$
\left\|x+x^{*}(x) y\right\| \leq\|x\|+\left|x^{*}(x)\right| \cdot\|y\| \leq 2-\frac{\varepsilon}{2} .
$$

The equivalence $(i) \Leftrightarrow($ iii $)$ can be proved in a very similar fashion to $(i) \Leftrightarrow$ (ii) using the fact that the norms of an operator and of its adjoint coincide.

Lemma 2.4. For $A \subset S_{X^{*}}$ the following assertions are equivalent:

(i) $X$ star-denies the Daugavet property with $A$.

(ii) There is an $\varepsilon>0$ such that for every $x^{*} \in A$ a vector $y \in S_{X}$ may be chosen so that every $x \in S\left(B_{X}, x^{*}, \varepsilon\right)$ fulfills $\|x+y\|<2-\varepsilon$.

(iii) There is an $\varepsilon>0$ such that for every $x^{*} \in A$ a vector $y \in S_{X}$ may be chosen so that every $y^{*} \in S\left(B_{X^{*}}, y, \varepsilon\right)$ fulfills $\left\|x^{*}+y^{*}\right\|<2-\varepsilon$.

One can prove Lemma 2.4 the same way as Lemma 2.3 . The following two lemmas form the main result of this section.

Lemma 2.5. Let there exist $\delta>0$ and $z^{*} \in S_{X^{*}}$ such that $X$ denies the Daugavet property with $S\left(B_{X}, z^{*}, \delta\right) \cap S_{X}$. Then $X$ is not a Daugavet domain.

Proof. According to Definition 1.1 we must prove that any $G: X \rightarrow Y$ is not a Daugavet center for any $Y$. It is easy to see that if $G$ is a Daugavet center then $G /\|G\|$ is as well, so we consider only the case $\|G\|=1$.

Take the $\varepsilon$ from item (ii) of Lemma 2.3. At first we show that if every $z \in S\left(B_{X}, z^{*}, \delta\right)$ satisfies $\|G z\| \leq 1-\varepsilon / 2$ then $G$ is not a Daugavet center. 
Put $\varepsilon_{0}:=\min \{\varepsilon / 2, \delta\}$, then for every $y \in S_{Y}$ and every $z \in S\left(B_{X}, z^{*}, \varepsilon_{0}\right)$ we have

$$
\|y+G z\| \leq 1+\|G z\| \leq 2-\frac{\varepsilon}{2} \leq 2-\varepsilon_{0} .
$$

Theorem 1.2, item (ii) implies that $G$ is not a Daugavet center.

So, we suppose that there is a $z_{0} \in S\left(B_{X}, z^{*}, \delta\right)$ with

$$
\left\|G z_{0}\right\|>1-\frac{\varepsilon}{2}
$$

We can assume $\left\|z_{0}\right\|=1$, because if $z_{0} \in B_{X}$ fulfills $z^{*}\left(z_{0}\right)>1-\delta$ and (2.2) then $z_{0} /\left\|z_{0}\right\|$ does as well. In addition, (2.2) implies that there is a $y_{0} \in$ $S_{Y}$ with $\left\|y_{0}-G z_{0}\right\|<\varepsilon / 2$. Since $X$ denies the Daugavet property with $S\left(B_{X}, z^{*}, \delta\right) \cap S_{X}$, there is an $x^{*} \in S_{X^{*}}$ such that every $x \in S\left(B_{X}, x^{*}, \varepsilon\right)$ satisfies $\left\|x+z_{0}\right\|<2-\varepsilon$. Hence for every $x \in S\left(B_{X}, x^{*}, \varepsilon\right)$ we have

$$
\left\|y_{0}+G x\right\| \leq\left\|y_{0}-G z_{0}\right\|+\left\|G x+G z_{0}\right\|<\frac{\varepsilon}{2}+\left\|x+z_{0}\right\|<2-\frac{\varepsilon}{2} .
$$

By Theorem 1.2, item (ii) $G$ is not a Daugavet center.

Lemma 2.6. Let there exist $\delta>0$ and $z \in S_{X}$ such that $X$ star-denies the Daugavet property with $S\left(B_{X^{*}}, z, \delta\right) \cap S_{X^{*}}$. Then $X$ is not a Daugavet range.

Using item (iii) of Lemma 2.4 and item (iii) of Theorem 1.2 one can prove Lemma 2.6 in a very similar fashion to Lemma 2.5.

\section{Two-dimensional lattices Denying the positive Daugavet PROPERTY}

Definition 3.1. We say that $F$ denies the positive Daugavet property with $A \subset S_{F}^{+}$if there is an $\varepsilon>0$ such that for every $a \in A$ there exists an $f^{*} \in S_{F^{*}}^{+}$satisfying

$$
\left\|\operatorname{Id}+f^{*} \otimes a\right\|<2-\varepsilon
$$

Definition 3.2. We say that $F$ star-denies the positive Daugavet property with $A \subset S_{F^{*}}^{+}$if there is an $\varepsilon>0$ such that for every $f^{*} \in A$ there exists an $a \in S_{F}^{+}$satisfying (3.1).

The following two lemmas are complete analogs of Lemmas 2.3 and 2.4 . so we skip their proofs.

Lemma 3.3. For $A \subset S_{F}^{+}$the following assertions are equivalent:

(i) $F$ denies the positive Daugavet property with $A$.

(ii) There is an $\varepsilon>0$ such that for every $a \in A$ a functional $f^{*} \in S_{F^{*}}^{+}$ may be chosen so that every $b \in S\left(B_{F}, f^{*}, \varepsilon\right) \cap B_{F}^{+}$fulfills $\|a+b\|<$ $2-\varepsilon$.

(iii) There is an $\varepsilon>0$ such that for every $a \in A$ a functional $f^{*} \in$ $S_{F^{*}}^{+}$may be chosen so that every $g^{*} \in S\left(B_{F^{*}}, a, \varepsilon\right) \cap B_{F^{*}}^{+}$fulfills $\left\|f^{*}+g^{*}\right\|<2-\varepsilon$. 
Lemma 3.4. For $A \subset S_{F^{*}}^{+}$the following assertions are equivalent:

(i) F star-denies the positive Daugavet property with $A$.

(ii) There is an $\varepsilon>0$ such that for every $f^{*} \in A$ a vector $a \in S_{F}^{+}$may be chosen so that every $b \in S\left(B_{F}, f^{*}, \varepsilon\right) \cap B_{F}^{+}$fulfills $\|a+b\|<2-\varepsilon$.

(iii) There is an $\varepsilon>0$ such that for every $f^{*} \in A$ a vector $a \in S_{F}^{+}$may be chosen so that every $g^{*} \in S\left(B_{F^{*}}, a, \varepsilon\right) \cap B_{F^{*}}^{+}$fulfills $\left\|f^{*}+g^{*}\right\|<2-\varepsilon$.

Recall that $F^{*}=\mathbb{R}^{2}$ with the norm

$$
\left\|\left(f_{1}, f_{2}\right)\right\|_{F^{*}}:=\max _{\left(a_{1}, a_{2}\right) \in B_{F}}\left|f_{1} a_{1}+f_{2} a_{2}\right|
$$

and $F^{* *}=F$. We introduce an order on $F^{*}$ the same way as on $F$. It is easy to see that $\|(1,0)\|_{F^{*}}=\|(0,1)\|_{F^{*}}=1$ and $\left\|\left(f_{1}, f_{2}\right)\right\|_{F^{*}}=\left\|\left(\left|f_{1}\right|,\left|f_{2}\right|\right)\right\|_{F^{*}}$ for every $\left(f_{1}, f_{2}\right) \in F^{*}$. Hence $F^{*}$ is a two-dimensional lattice as well. Lemmas 3.3 and 3.4 evidently imply the following fact (which one can easily deduce from Definitions 3.1 and 3.2 as well).

Lemma 3.5. Let $A \subset S_{F}^{+}$and $\tilde{A} \subset S_{F^{*}}^{+}$.

(a) If $F$ denies the positive Daugavet property with $A$ then $F^{*}$ stardenies the positive Daugavet property with A.

(b) If $F$ star-denies the positive Daugavet property with $\tilde{A}$ then $F^{*}$ denies the positive Daugavet property with $\tilde{A}$.

Here is the key lemma of this section. In its proof we use the idea from Theorem 5.1 of [1].

Lemma 3.6. Let there exist $w^{*} \in S_{F^{*}}^{+}$and $\delta>0$ such that $F$ denies the positive Daugavet property with $S\left(B_{F}, w^{*}, \delta\right) \cap S_{F}^{+}$. Then $X_{1} \oplus_{F} X_{2}$ is not a Daugavet domain for any $X_{1}$ and $X_{2}$.

Proof. It is easy to see that $\left(X_{1} \oplus_{F} X_{2}\right)^{*}=X_{1}^{*} \oplus_{F^{*}} X_{2}^{*}$ for every $X_{1}$ and $X_{2}$. Pick a $z^{*}=\left(z_{1}^{*}, z_{2}^{*}\right) \in S_{\left(X_{1} \oplus_{F} X_{2}\right)^{*}}$ with $\left(\left\|z_{1}^{*}\right\|,\left\|z_{2}^{*}\right\|\right)=w^{*}$. Then for a $y=\left(y_{1}, y_{2}\right) \in S\left(B_{X_{1} \oplus_{F} X_{2}}, z^{*}, \delta\right) \cap S_{X_{1} \oplus_{F} X_{2}}$ we have

$$
\left\|z_{1}^{*}\right\|\left\|y_{1}\right\|+\left\|z_{2}^{*}\right\|\left\|y_{2}\right\| \geq z_{1}^{*}\left(y_{1}\right)+z_{2}^{*}\left(y_{2}\right)=z^{*}(y)>1-\delta .
$$

Hence $a:=\left(\left\|y_{1}\right\|,\left\|y_{2}\right\|\right) \in S\left(B_{F}, w^{*}, \delta\right) \cap S_{F}^{+}$. By item (ii) of Lemma 3.3 there exist $\varepsilon>0$ and $f^{*} \in S_{F^{*}}^{+}$such that every $b \in S\left(B_{F}, f^{*}, \varepsilon\right) \cap B_{F}^{+}$satisfies $\|a+b\|<2-\varepsilon$.

Pick an $x^{*}=\left(x_{1}^{*}, x_{2}^{*}\right) \in S_{\left(X_{1} \oplus_{F} X_{2}\right)^{*}}$ with $\left(\left\|x_{1}^{*}\right\|,\left\|x_{2}^{*}\right\|\right)=f^{*}$. Then for every $x=\left(x_{1}, x_{2}\right) \in S\left(B_{X_{1} \oplus_{F} X_{2}}, x^{*}, \varepsilon\right)$ we have $b_{x}:=\left(\left\|x_{1}\right\|,\left\|x_{2}\right\|\right) \in$ $S\left(B_{F}, f^{*}, \varepsilon\right) \cap B_{F}^{+}$and therefore

$$
\begin{aligned}
\|x+y\| & =\left\|\left(\left\|x_{1}+y_{1}\right\|,\left\|x_{2}+y_{2}\right\|\right)\right\| \\
& \leq\left\|\left(\left\|x_{1}\right\|+\left\|y_{1}\right\|,\left\|x_{2}\right\|+\left\|y_{2}\right\|\right)\right\|=\left\|a+b_{x}\right\|<2-\varepsilon .
\end{aligned}
$$

By item (ii) of Lemma $2.3 X_{1} \oplus_{F} X_{2}$ denies the Daugavet property for $S\left(B_{X_{1} \oplus_{F} X_{2}}, z^{*}, \delta\right) \cap S_{X_{1} \oplus_{F} X_{2}}$. So, Lemma 2.5 implies that $X_{1} \oplus_{F} X_{2}$ is not a Daugavet domain. 
The same conclusions based on item (iii) of Lemma 3.4, item (iii) of Lemma 2.4. and Lemma 2.6 prove the following fact:

Lemma 3.7. Let there exist $w \in S_{F}^{+}$and $\delta>0$ such that $F$ star-denies the positive Daugavet property with $S\left(B_{F^{*}}, w, \delta\right) \cap S_{F^{*}}^{+}$. Then $X_{1} \oplus_{F} X_{2}$ is not a Daugavet range for any $X_{1}$ and $X_{2}$.

\section{Sums of spaces Which are not Daugavet Ranges}

In this section we find a large class of those $F$ which star-deny the positive Daugavet property with some $S\left(B_{F^{*}}, w, \delta\right) \cap S_{F^{*}}^{+}$. Throughout this and the following sections $e_{1}:=(1,0) \in S_{F}^{+}, e_{2}:=(0,1) \in S_{F}^{+}$, and the symbol $[a, b]$ is reserved for the line segment with the end points in $a, b \in F$.

Lemma 4.1. Let $D$ be a closed subset of $S_{F^{*}}^{+}$. Suppose for every $f^{*} \in D$ there exists an $\varepsilon>0$ such that the property $P\left(f^{*}, \varepsilon\right):=\left\{\right.$ there is an $a \in S_{F}^{+}$ such that every $b \in S\left(B_{F}, f^{*}, \varepsilon\right) \cap B_{F}^{+}$satisfies $\left.\|a+b\|<2-\varepsilon\right\}$ holds true. Then $F$ star-denies the positive Daugavet property with $D$.

Proof. Note that if $P\left(f^{*}, \varepsilon\right)$ holds true then $P\left(f^{*}, \varepsilon_{1}\right)$ holds for every $\varepsilon_{1}$ : $0<\varepsilon_{1}<\varepsilon$. Our goal is to show that there exists a common $\varepsilon_{\min }>0$ such that $P\left(f^{*}, \varepsilon_{\text {min }}\right)$ holds true for every $f^{*} \in D$.

Consider the function $u\left(f^{*}\right): D \rightarrow(0,1), u\left(f^{*}\right)=\sup \{\varepsilon>0$ : $P\left(f^{*}, \varepsilon\right)$ holds true $\}$. Let us prove that $u\left(f^{*}\right)$ reaches its minimum value on $D$. Since $D$ is compact, it is sufficient to show that $u\left(f^{*}\right)$ is lower semicontinuous, i.e. that the set $u^{-1}((x, 1))$ is open for every $x \in[0,1)$.

Let $f^{*} \in u^{-1}((x, 1))$. This means that $u\left(f^{*}\right)=\sup \{\varepsilon>0$ : $P\left(f^{*}, \varepsilon\right)$ holds true $\}>x$. Hence there exist $\varepsilon_{0}>x$ and $a \in S_{F}^{+}$such that every $b \in S\left(B_{F}, f^{*}, \varepsilon_{0}\right) \cap B_{F}^{+}$fulfills $\|a+b\|<2-\varepsilon_{0}$.

Take an $\varepsilon_{1}: x<\varepsilon_{1}<\varepsilon_{0}$ and put $\delta:=\varepsilon_{0}-\varepsilon_{1}$. The set $D \cap B_{F^{*}}\left(f^{*}, \delta\right)$ is a relative neighborhood of $f^{*}$ in $D$. Let us show that $D \cap B_{F^{*}}\left(f^{*}, \delta\right) \subset$ $u^{-1}((x, 1))$.

Let $f_{1}^{*} \in D \cap B_{F^{*}}\left(f^{*}, \delta\right)$. Then every $b \in S\left(B_{F}, f_{1}^{*}, \varepsilon_{1}\right) \cap B_{F}^{+}$fulfills

$$
f^{*}(b) \geq f_{1}^{*}(b)-\delta>1-\varepsilon_{1}-\delta=1-\varepsilon_{0} .
$$

Thus $b \in S\left(B_{F}, f^{*}, \varepsilon_{0}\right) \cap B_{F}^{+}$, so we have

$$
\|a+b\|<2-\varepsilon_{0}<2-\varepsilon_{1} .
$$

This means that $u\left(f_{1}^{*}\right) \geq \varepsilon_{1}>x$ and $f_{1}^{*} \in u^{-1}((x, 1))$. Consequently, $u^{-1}((x, 1))$ is open and $u\left(f^{*}\right)$ is lower semicontinuous. Then there exists an $f_{0}^{*} \in D$ such that

$$
u\left(f_{0}^{*}\right)=\min _{f^{*} \in D} u\left(f^{*}\right) .
$$

Take an $\varepsilon_{\text {min }}: 0<\varepsilon_{\min }<u\left(f_{0}^{*}\right)$ then $P\left(f^{*}, \varepsilon_{\text {min }}\right)$ holds true for every $f^{*} \in D$. 
Lemma 4.2. Let $a \in S_{F}^{+}$and $f^{*} \in S_{F^{*}}^{+}$. Suppose for every $\varepsilon>0$ there is $a b \in S\left(B_{F}, f^{*}, \varepsilon\right) \cap B_{F}^{+}$with $\|a+b\| \geq 2-\varepsilon$. Then there exists a $b_{0} \in S_{F}^{+}$ such that $f^{*}\left(b_{0}\right)=1$ and $\left[a, b_{0}\right] \subset S_{F}^{+}$.

Proof. Consider a vanishing sequence $\left\{\varepsilon_{n}\right\}_{n=1}^{\infty}, \varepsilon_{n}>0$. For every $n \in \mathbb{N}$ there exists a $b_{n} \in B_{F}^{+}$with $f^{*}\left(b_{n}\right)>1-\varepsilon_{n}$ and $\left\|a+b_{n}\right\| \geq 2-\varepsilon_{n}$.

Since $B_{F}^{+}$is a compact set, there exists a subsequence $\left\{b_{n_{i}}\right\}_{i=1}^{\infty}$ of $\left\{b_{n}\right\}_{n=1}^{\infty}$ that converges to some $b_{0} \in B_{F}^{+}$. Then $f^{*}\left(b_{0}\right)=1$ and $\left\|a+b_{0}\right\|=2$ which implies $\left[a, b_{0}\right] \subset S_{F}^{+}$.

Denote $\mathfrak{N}_{3}$ the class of those $F$ whose $S_{F}^{+}$is a polygon which consists of at most three edges.

Lemma 4.3. Let $F \notin \mathfrak{N}_{3}$. Then $F$ star-denies the positive Daugavet property with $S_{F^{*}}^{+}$.

Proof. Assume to the contrary that there exists an $f^{*} \in S_{F^{*}}^{+}$such that for every $\varepsilon>0$ and $a_{0} \in S_{F}^{+}$there is a $b \in S\left(B_{F}, f^{*}, \varepsilon\right) \cap B_{F}^{+}$with $\left\|a_{0}+b\right\| \geq 2-\varepsilon$.

Consider the set $\Delta:=\left\{a \in S_{F}^{+}: f^{*}(a)=1\right\}$. It is easy to see that $\Delta$ is a segment or a point. Put $a_{0}:=e_{1}$. By Lemma 4.2 there exists a $b_{0} \in \Delta$ such that $\left[b_{0}, e_{1}\right] \subset S_{F}^{+}$. If we put $a_{0}:=e_{2}$ we obtain a $b_{1} \in \Delta$ with $\left[b_{1}, e_{2}\right] \subset S_{F}^{+}$. Then $F \in \mathfrak{N}_{3}$, because $S_{F}^{+}$consists of at most three segments: $\left[b_{0}, e_{1}\right], \Delta$ and $\left[b_{1}, e_{2}\right]$. This contradiction completes the proof.

Lemma 4.4. Let $S_{F}^{+}$be a polygon which consists of exactly three edges. Then there exists a $w^{*}=\left(w_{1}, w_{2}\right) \in S_{F^{*}}^{+}$with $w_{1}<1$ and $w_{2}<1$ such that $F$ star-denies the positive Daugavet property with $S_{F^{*}}^{+} \backslash \stackrel{\circ}{B}_{F^{*}}\left(w^{*}, \delta_{0}\right)$ for every $\delta_{0}>0$.

Proof. Since $S_{F}^{+}$consists of three edges, it has four vertexes. The points $e_{1}$ and $e_{2}$ are two of them, denote $h_{1}$ and $h_{2}$ the remaining ones in such a way that $\left[e_{1}, h_{1}\right] \cup\left[e_{2}, h_{2}\right] \subset S_{F}^{+}$. There is the unique $w^{*}=\left(w_{1}, w_{2}\right) \in S_{F^{*}}^{+}$such that $\left\{a \in S_{F}^{+}: w^{*}(a)=1\right\}=\left[h_{1}, h_{2}\right]$. It is obvious that $w_{1}<1$ and $w_{2}<1$.

Consider a $\delta_{0}>0$ and an $f^{*} \in S_{F^{*}}^{+} \backslash \stackrel{\circ}{B}_{F^{*}}\left(w^{*}, \delta_{0}\right)$. Denote $\Delta:=\{a \in$ $\left.S_{F}^{+}: f^{*}(a)=1\right\}$, it is a segment or a point. Assume that for every $\varepsilon>0$ and $a \in S_{F}^{+}$there exists a $b \in S\left(B_{F}, f^{*}, \varepsilon\right) \cap B_{F}^{+}$with $\|a+b\| \geq 2-\varepsilon$. By Lemma 4.2 there are $b_{1}, b_{2} \in \Delta$ such that $\left[b_{1}, e_{1}\right] \subset S_{F}^{+}$and $\left[b_{2}, e_{2}\right] \subset S_{F}^{+}$. Hence $b_{1} \in\left[e_{1}, h_{1}\right]$ and $b_{2} \in\left[e_{2}, h_{2}\right]$. Since $\left[e_{1}, h_{1}\right] \cap\left[e_{2}, h_{2}\right]=\emptyset$ then $\Delta \nsubseteq$ $\left[e_{1}, h_{1}\right], \Delta \nsubseteq\left[e_{2}, h_{2}\right]$, and $\Delta$ is a segment. Consequently, $\Delta \subset\left[h_{1}, h_{2}\right]$. But then $w^{*}=f^{*}$, so we come to contradiction.

Thus for every $f^{*} \in S_{F^{*}}^{+} \backslash \stackrel{\circ}{B}_{F^{*}}\left(w^{*}, \delta_{0}\right)$ there are $\varepsilon>0$ and $a \in S_{F}^{+}$ such that every $b \in S\left(B_{F}, f^{*}, \varepsilon\right) \cap B_{F}^{+}$satisfies $\|a+b\|<2-\varepsilon$. Since $S_{F^{*}}^{+} \backslash \stackrel{\circ}{B}_{F^{*}}\left(w^{*}, \delta_{0}\right)$ is closed, Lemma 4.1 implies the needed result.

Denote $\mathfrak{N}_{2}$ the class of those $F$ whose $S_{F}^{+}$is a polygon which consists of at most two edges. 
Corollary 4.5. Let $F \notin \mathfrak{N}_{2}$. Then there is a $\delta>0$ such that $F$ star-denies the positive Daugavet property with $S\left(B_{F^{*}}, e_{1}, \delta\right) \cap S_{F^{*}}^{+}$.

Proof. If $F \notin \mathfrak{N}_{3}$ then by Lemma 4.3 the statement is proved.

If $S_{F}^{+}$is a polygon which consists of exactly three edges then by Lemma4.4 there exists a $w^{*}=\left(w_{1}, w_{2}\right) \in S_{F^{*}}^{+}$with $w_{1}<1$ such that $F$ star-denies the positive Daugavet property with $S_{F^{*}}^{+} \backslash \stackrel{\circ}{B}_{F^{*}}\left(w^{*}, \delta_{0}\right)$ for every $\delta_{0}>0$. Pick a $\delta_{0}>0$ with $\delta_{0}+w_{1}<1$ and a $\delta$ such that $0<\delta<1-w_{1}-\delta_{0}$. Then every $f^{*} \in \stackrel{\circ}{B}_{F^{*}}\left(w^{*}, \delta_{0}\right)$ satisfies

$$
f^{*}\left(e_{1}\right)<w^{*}\left(e_{1}\right)+\delta_{0}=w_{1}+\delta_{0}<1-\delta .
$$

Hence $\stackrel{\circ}{B}_{F^{*}}\left(w^{*}, \delta_{0}\right) \cap S\left(B_{F^{*}}, e_{1}, \delta\right)=\emptyset$. Thus $F$ star-denies the positive Daugavet property with $S\left(B_{F^{*}}, e_{1}, \delta\right) \cap S_{F^{*}}^{+}$.

We obtain the following fact by the successive application of Corollary 4.5 and Lemma 3.7.

Corollary 4.6. Let $F \notin \mathfrak{N}_{2}$. Then $X_{1} \oplus_{F} X_{2}$ is not a Daugavet range for any $X_{1}$ and $X_{2}$.

\section{Sums of SPACES Which ARE NOt DAUGAVET DOMAINS}

Lemma 5.1. Let $F^{*} \notin \mathfrak{N}_{2}$. Then $X_{1} \oplus_{F} X_{2}$ is not a Daugavet domain for any $X_{1}$ and $X_{2}$.

Proof. By Corollary 4.5 there is a $\delta>0$ such that $F^{*}$ star-denies the positive Daugavet property with $S\left(B_{F^{* *}}, e_{1}, \delta\right) \cap S_{F^{* *}}^{+}$. Recall that $F^{* *}=F$. Therefore it follows from Lemma 3.5 that $F$ denies the positive Daugavet property with $S\left(B_{F}, e_{1}, \delta\right) \cap S_{F}^{+}$. Then Lemma 3.6 gives the needed result.

We characterize the class of those $F$ such that $S_{F^{*}}^{+}$is a polygon with at most two edges, with the help of the following notation. Consider an $F$ whose $S_{F}^{+}$is a polygon with $n$ edges. Denote $\hat{x}_{1}:=\max _{(1, y) \in S_{F}^{+}} y$ and $\hat{x}_{2}:=\max _{(x, 1) \in S_{F}^{+}} x$. We say that $F$ belongs to $\mathcal{F}_{n-1, n}$ if $\hat{x}_{1}>0$ and $\hat{x}_{2}>0$. If only one of $\hat{x}_{j}$ equals zero, we say that $F \in \mathcal{F}_{n, n}$. And if both $\hat{x}_{1}=\hat{x}_{2}=0$ then $F \in \mathcal{F}_{n+1, n}$ (see Figure 10).

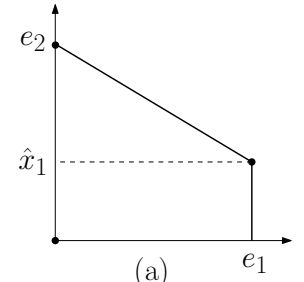

(a)

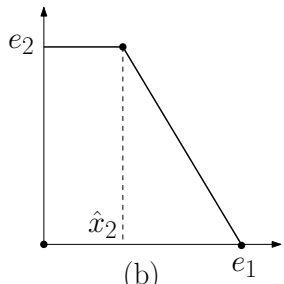

(b)

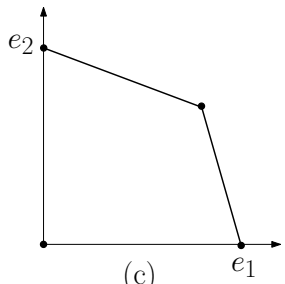

(c)

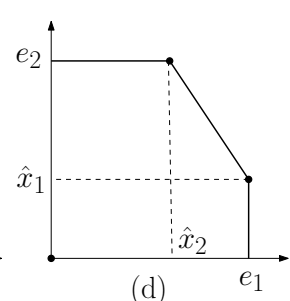

(d)

Figure 1. Those $F$ whose $S_{F}^{+}$are presented on pictures (a) and (b), belong to $\mathcal{F}_{2,2}$. Picture (c) shows $S_{F}^{+}$of $F \in \mathcal{F}_{3,2}$ and (d) shows $S_{F}^{+}$of $F \in \mathcal{F}_{2,3}$. 
Thus, $\mathfrak{N}_{2}=\left\{\ell_{1}^{(2)}\right\} \cup\left\{\ell_{\infty}^{(2)}\right\} \cup \mathcal{F}_{2,2} \cup \mathcal{F}_{3,2}$. Let $n \in \mathbb{N}$ and $m \in\{n-1, n, n+1\}$. It is easy to see that $F^{*} \in \mathcal{F}_{n, m}$ if and only if $F \in \mathcal{F}_{m, n}$. Therefore, if $F^{*} \in \mathcal{F}_{2,2} \cup \mathcal{F}_{3,2}$ then $F \in \mathcal{F}_{2,2} \cup \mathcal{F}_{2,3}$. So, we obtain the following fact:

Corollary 5.2. Let $F \notin\left\{\ell_{1}^{(2)}\right\} \cup\left\{\ell_{\infty}^{(2)}\right\} \cup \mathcal{F}_{2,2} \cup \mathcal{F}_{2,3}=: \mathfrak{M}_{2}$. Then $X_{1} \oplus_{F} X_{2}$ is not a Daugavet domain for any $X_{1}$ and $X_{2}$.

\section{Examples of Daugavet Centers acting From and into a Sum OF TWO BANACH SPACES}

In this section we show that for every $F \in \mathfrak{M}_{2}$ there exists a Daugavet domain $X_{1} \oplus_{F} X_{2}$, and for every $F \in \mathfrak{N}_{2}$ there is a Daugavet range $X_{1} \oplus_{F} X_{2}$.

For $F=\ell_{1}^{(2)}$ and $F=\ell_{\infty}^{(2)}$ several examples of $X_{1} \oplus_{F} X_{2}$ which are Daugavet domains and Daugavet ranges, are known. For instance, if $X$ is a Daugavet domain then for every $E$ the sum $X \oplus_{\infty} E$ is as well; and if $X$ is a Daugavet range then $X \oplus_{1} E$ is. If $G_{1}: X_{1} \rightarrow Y_{1}$ and $G_{2}: X_{2} \rightarrow Y_{2}$ are Daugavet centers then $G: X_{1} \oplus_{1} X_{2} \rightarrow Y_{1} \oplus_{1} Y_{2}$ and $\tilde{G}: X_{1} \oplus_{\infty} X_{2} \rightarrow Y_{1} \oplus_{\infty} Y_{2}$ which map every $\left(x_{1}, x_{2}\right)$ into $\left(G_{1} x_{1}, G_{2} x_{2}\right)$, are Daugavet centers as well [3].

For future reference we mention the following fact:

Lemma 6.1 (6], Lemma 2.8). If $X$ has the Daugavet property then for every finite-dimensional subspace $Y_{0}$ of $X$, every $\varepsilon>0$, and every slice $S\left(B_{X}, x^{*}, \varepsilon\right)$ there is an $x \in S\left(B_{X}, x^{*}, \varepsilon\right)$ such that every $y \in Y_{0}$ and $t \in \mathbb{R}$ fulfill

$$
\|y+t x\| \geq(1-\varepsilon)(\|y\|+|t|) .
$$

Consider an $F \in \mathcal{F}_{2,2} \cup \mathcal{F}_{2,3}$. Denote $c_{1}:=\left(1, \hat{x}_{1}\right) \in S_{F}^{+}$and $c_{2}:=\left(\hat{x}_{2}, 1\right) \in$ $S_{F}^{+}$. Then $\left[c_{1}, c_{2}\right] \subset S_{F}^{+}$(see Figure 2 ).

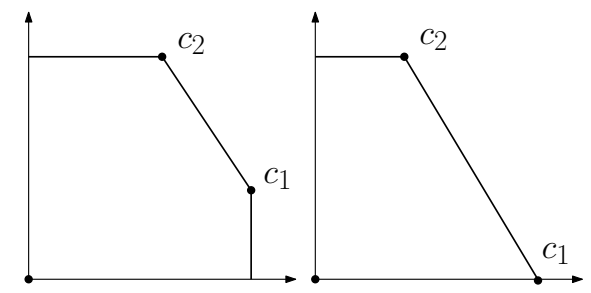

(a)

(b)

Figure 2. Picture (a) shows $S_{F}^{+}$of $F \in \mathcal{F}_{2,3}$ and (b) presents $S_{F}^{+}$of $F \in \mathcal{F}_{2,2}$.

Let the line containing $\left[c_{1}, c_{2}\right]$ have the equation $f_{1} a_{1}+f_{2} a_{2}=1$ with $\left(f_{1}, f_{2}\right) \in S_{F^{*}}^{+}$. Remark that for every $w^{*} \in S_{F^{*}}^{+}$and $\varepsilon>0$ we have $S\left(B_{F}, w^{*}, \varepsilon\right) \cap\left[c_{1}, c_{2}\right] \neq \emptyset$. In other words, there exists an $\left(a_{1}, a_{2}\right) \in$ $S\left(B_{F}, w^{*}, \varepsilon\right)$ such that $f_{1} a_{1}+f_{2} a_{2}=1$. 
Proposition 6.2. Let $X$ have the Daugavet property, $F \in \mathcal{F}_{2,2} \cup \mathcal{F}_{2,3}$, and let $\left(f_{1}, f_{2}\right) \in S_{F^{*}}^{+}$be the functional described above. Then $G: X \oplus_{F} X \rightarrow X$, $G\left(x_{1}, x_{2}\right)=f_{1} x_{1}+f_{2} x_{2}$ is a Daugavet center.

Proof. At first, calculate $\|G\|$ :

$$
\|G\|=\sup _{\left(x_{1}, x_{2}\right) \in S_{X \oplus_{F} X}}\left\|f_{1} x_{1}+f_{2} x_{2}\right\|=\sup _{\left(x_{1}, x_{2}\right) \in S_{X \oplus_{F} X}}\left(f_{1}\left\|x_{1}\right\|+f_{2}\left\|x_{2}\right\|\right)=1 .
$$

Let $\varepsilon>0, y_{0} \in S_{X}$ and $x^{*}=\left(x_{1}^{*}, x_{2}^{*}\right) \in S_{\left(X \oplus_{F} X\right)^{*}}$.

By Lemma 6.1 there exists an $\tilde{x}_{1} \in B_{X}$ with $x_{1}^{*}\left(\tilde{x}_{1}\right) \geq\left\|x_{1}^{*}\right\|(1-\varepsilon / 4)$ and

$$
\left\|y_{0}+t \tilde{x}_{1}\right\| \geq\left(1-\frac{\varepsilon}{4}\right)(1+|t|)
$$

for every $t \in \mathbb{R}$. Using again Lemma 6.1 we have an $\tilde{x}_{2} \in B_{X}$ with $x_{2}^{*}\left(\tilde{x}_{2}\right) \geq$ $\left\|x_{2}^{*}\right\|(1-\varepsilon / 4)$ and

$$
\left\|y+t \tilde{x}_{2}\right\| \geq\left(1-\frac{\varepsilon}{4}\right)(\|y\|+|t|)
$$

for every $y \in \operatorname{lin}\left\{y_{0}, \tilde{x}_{1}\right\}$ and every $t \in \mathbb{R}$.

Denote $w^{*}:=\left(\left\|x_{1}^{*}\right\|,\left\|x_{2}^{*}\right\|\right) \in S_{F^{*}}^{+}$. Let $\left(a_{1}, a_{2}\right) \in S\left(B_{F}, w^{*}, 3 \varepsilon / 4\right)$ such that $f_{1} a_{1}+f_{2} a_{2}=1$. Then for $x:=\left(a_{1} \tilde{x}_{1}, a_{2} \tilde{x}_{2}\right) \in B_{X \oplus_{F} X}$ we have

$$
\begin{aligned}
x^{*}(x)=a_{1} x_{1}^{*}\left(\tilde{x}_{1}\right)+a_{2} x_{2}^{*}\left(\tilde{x}_{2}\right) & \geq\left(1-\frac{\varepsilon}{4}\right)\left(a_{1}\left\|x_{1}^{*}\right\|+a_{2}\left\|x_{2}^{*}\right\|\right) \\
& \geq\left(1-\frac{\varepsilon}{4}\right)\left(1-\frac{3 \varepsilon}{4}\right)>1-\varepsilon .
\end{aligned}
$$

Hence $x \in S\left(B_{X \oplus_{F} X}, x^{*}, \varepsilon\right)$ and

$$
\left\|y_{0}+G x\right\|=\left\|y_{0}+f_{1} a_{1} \tilde{x}_{1}+f_{2} a_{2} \tilde{x}_{2}\right\|
$$

by $(6.2)$

$$
>\left(1-\frac{\varepsilon}{4}\right)\left(\left\|y_{0}+f_{1} a_{1} \tilde{x}_{1}\right\|+f_{2} a_{2}\right)
$$

by (6.1)

$$
>\left(1-\frac{\varepsilon}{4}\right)^{2}\left(1+f_{1} a_{1}+f_{2} a_{2}\right)=2\left(1-\frac{\varepsilon}{4}\right)^{2}>2-\varepsilon .
$$

Theorem 1.2, item (ii) implies that $G$ is a Daugavet center.

Corollary 6.3. For an $F$ there exists a Daugavet domain $X_{1} \oplus_{F} X_{2}$ if and only if $F \in \mathfrak{M}_{2}$.

Remark 6.4. Note that $\mathfrak{M}_{2} \nsubseteq \mathfrak{N}_{2}$. Then Corollary 6.3 and Corollary 4.6 imply that there exist Daugavet domains which are not Daugavet ranges.

Now we present more examples of Daugavet centers acting from $X_{1} \oplus_{F} X_{2}$ where $F=\ell_{1}^{(2)}$ or $F=\ell_{\infty}^{(2)}$.

Proposition 6.5. Let $X$ have the Daugavet property. Then

(a) The operator $G$ : $X \oplus_{1} X \rightarrow X, G\left(x_{1}, x_{2}\right)=x_{1}+x_{2}$ is a Daugavet center. 
(b) For every $f_{1}, f_{2}>0$ the operator $G$ : $X \oplus_{\infty} X \rightarrow X, G\left(x_{1}, x_{2}\right)=$ $f_{1} x_{1}+f_{2} x_{2}$ is a Daugavet center.

Proposition 6.5 can be proved the same way as Proposition 6.2.

Proposition 6.6. Let $X$ have the Daugavet property, $F \in \mathcal{F}_{2,2} \cup \mathcal{F}_{3,2}$, and let $\left(f_{1}, f_{2}\right) \in S_{F}^{+}$be the vector described above. Then $G: X \rightarrow X \oplus_{F} X$, $G x=\left(f_{1} x, f_{2} x\right)$ is a Daugavet center.

Proof. Consider the adjoint operator $G^{*}: X^{*} \oplus_{F^{*}} X^{*} \rightarrow X^{*}$. For every $\left(x_{1}^{*}, x_{2}^{*}\right) \in X^{*} \oplus_{F^{*}} X^{*}$ and every $x \in X$ we have

$$
G^{*}\left(x_{1}^{*}, x_{2}^{*}\right)(x)=\left\langle\left(f_{1} x, f_{2} x\right),\left(x_{1}^{*}, x_{2}^{*}\right)\right\rangle=f_{1} x_{1}^{*}(x)+f_{2} x_{2}^{*}(x) .
$$

Consequently, $G^{*}\left(x_{1}^{*}, x_{2}^{*}\right)=f_{1} x_{1}^{*}+f_{2} x_{2}^{*}$ for every $\left(x_{1}^{*}, x_{2}^{*}\right) \in X^{*} \oplus_{F^{*}} X^{*}$. By Proposition $6.2 G^{*}$ is a Daugavet center. The equation (1.1) implies that if $G^{*}$ is a Daugavet center then $G$ is as well.

Corollary 6.7. For an $F$ there exists a Daugavet range $X_{1} \oplus_{F} X_{2}$ if and only if $F \in \mathfrak{N}_{2}$.

Remark 6.8. Since $\mathfrak{N}_{2} \nsubseteq \mathfrak{M}_{2}$, we have the examples of Daugavet ranges which are not Daugavet domains.

Proposition 6.9 which gives more examples of Daugavet centers acting into $X_{1} \oplus_{F} X_{2}$ for $F=\ell_{1}^{(2)}$ and $F=\ell_{\infty}^{(2)}$, can be proved in a very similar way to Proposition 6.6.

Proposition 6.9. Let $X$ have the Daugavet property. Then

(a) The operator $G$ : $X \rightarrow X \oplus_{\infty} X, G x=(x, x)$ is a Daugavet center.

(b) For every $f_{1}, f_{2}>0$ the operator $G$ : $X \rightarrow X \oplus_{1} X, G x=\left(f_{1} x, f_{2} x\right)$ is a Daugavet center.

\section{ACKNowledGements}

The author is grateful to her scientific adviser Vladimir M. Kadets for attention and numerous fruitful discussions, and to the referee for useful remarks.

\section{REFERENCES}

[1] Bilik D., Kadets V., Shvidkoy R., Werner D., Narrow operators and the Daugavet property for ultraproducts, Positivity, 2005, 9, 45-62

[2] Bourgain J., Rosenthal H.P., Martingales valued in certain subspaces of $L^{1}$, Israel J. Math., 1980, 37, 54-75

[3] Bosenko T., Kadets V., Daugavet centers, Zh. Mat. Fiz. Anal. Geom., (in press), preprint available at http://arxiv.org/abs/0910.4503

[4] Daugavet I.K., On a property of completely continuous operators in the space $C$, Uspekhi Mat. Nauk, 1963, 18, 157-158, (in Russian)

[5] Kadets V.M., Shepelska V., Werner D., Quotients of Banach spaces with the Daugavet property, Bull. Pol. Acad. Sci. Math., 2008, 56, 131-147

[6] Kadets V.M., Shvidkoy R.V., Sirotkin G.G., Werner D., Banach spaces with the Daugavet property, Trans. Amer. Math. Soc., 2000, 352, 855-873 
[7] Kadets V.M., Werner D., A Banach space with the Schur and the Daugavet property, Proc. Amer. Math. Soc., 2004, 132, 1765-1773

[8] Lindenstrauss J., Tzafriri L., Classical Banach Spaces II: Function spaces, SpringerVerlag, Berlin-Heidelberg-New York, 1979

[9] Lozanovskii G.Ya., On almost integral operators in KB-spaces, Vestnik Leningrad Univ. Mat. Mekh. Astr., 1966, 21, 35-44, (in Russian)

[10] Popov M.M., Daugavet type inequalities for narrow operators in the space $L_{1}$, Mat. Stud., 2003, 20, 75-84

[11] Shvidkoy R.V., Geometric aspects of the Daugavet property, J. Funct. Anal., 2000, $176,198-212$

[12] Talagrand M., The three-space problem for $L^{1}$, J. Amer. Math. Soc., 1990, 3, 9-29

[13] Werner D., Recent progress on the Daugavet property, Irish Math. Soc. Bull., 2001, 46, 77-97

[14] Werner D., The Daugavet equation for operators on function spaces, J. Funct. Anal., 1997, 143, 117-128

[15] Wojtaszczyk P., Some remarks on the Daugavet equation, Proc. Amer. Math. Soc., 1992, 115, 1047-1052

Department of Mechanics and Mathematics, V.N. Karazin Kharkiv National University, Pl. Svobody 4, 61077 Kharkiv, Ukraine

E-mail address: t.bosenko@mail.ru 